\title{
HANDLEBODY BUNDLES AND POLYTOPES
}

\author{
SEBASTIAN HENSEL AND DAWID KIELAK
}

\begin{abstract}
We construct examples of fibered three-manifolds with first Betti number at least 2 and with fibered faces all of whose monodromies extend to a handlebody.
\end{abstract}

\section{INTRODUCTION}

Suppose that $M$ is an orientable three-manifold which fibers over the circle with fiber a closed connected surface; let $\omega: \pi_{1}(M) \rightarrow \mathbb{Z}$ denote the induced homomorphism (we will say that $\omega$ is a fibered class in the first cohomology of $M$ ). Thurston [Thu86] developed a theory which describes all possible ways in which $M$ can fiber. Namely, he constructed a convex polytope $P_{M}$ in $H^{1}(M ; \mathbb{R})$ such that the fibered classes of $M$ are exactly the integral classes in cones over certain "fibered" faces of $P_{M}$.

In particular, all the integral classes $\omega^{\prime}$ in the cone $C_{F}$ containing the class $\omega$ are also fibered. For each such $\omega^{\prime}$, there is an associated monodromy homeomorphism (determined up to isotopy). As all of these monodromies describe the same 3-manifold, these elements of mapping class groups of different surfaces should share interesting properties - but is in general extremely hard to explicitly relate them.

In this article we present a construction of three-manifolds in which all of these monodromies share the property that they extend to handlebodies. Namely, we show:

Theorem 1.1. There exists infinitely many pairwise non-diffeomorphic, closed three-manifolds $M$ with $b_{1}(M) \geqslant 2$ and with the following property: the Thurston polytope $P_{M}$ of $M$ contains a fibered face $F$ such that every integral class in the cone $C_{F}$ over $F$ is fibered, and its associated monodromy extends from the closed surface on which it is defined to a handlebody.

The proof of this theorem relies on a connection of handlebody bundles to free-by-cyclic groups; the latter have recently been studied in analogy to fibered three-manifolds, see e.g. [DKL15, DKL17, DKL17b, FK18, Kie20, Mut]. Formally, Theorem 1.1 follows from Theorems 1.2 and 1.3 below.

To elucidate the connection, we need the following definition. We say that a class $\omega$ is compatible with a handlebody bundle if $\omega$ is induced by $M$ fibering over the circle with monodromy a mapping class $\varphi$ of some closed surface $S_{g}$, such that $\varphi$ extends to a mapping class of a handlebody $V_{g}$. We say that $\omega$

Date: March 23, 2020. 
is fully compatible with a handlebody bundle if in addition the inclusion map $M \hookrightarrow W$ induces an isomorphism $H_{1}(M ; \mathbb{Q}) \cong H_{1}(W ; \mathbb{Q})$ where $W$ denotes the fibered four-manifold whose monodromy is the extension of $\varphi$ to $V_{g}$.

The fundamental group of $W$ is a free-by-cyclic group $\Gamma=\pi_{1}(W)$ fitting into the following commutative diagram:

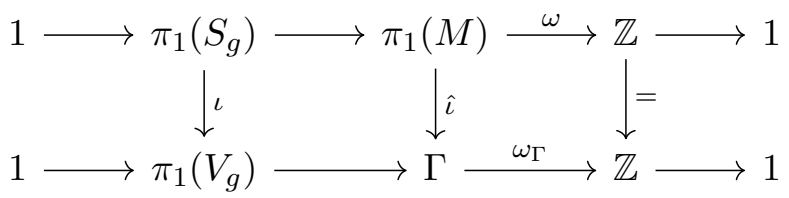

where $\omega_{\Gamma}$ is induced by $\omega$, and where $\iota$ and $\hat{\imath}$ are epimorphisms induced by the embeddings $S_{g} \hookrightarrow V_{g}$ and $M \hookrightarrow W$.

In recent work [Kie20], the second author constructed a convex polytope $P_{\Gamma}$ which serves as an analogue of the Thurston polytope $P_{M}$, classifying fiberings of $\Gamma$, i.e. maps $\Gamma \rightarrow \mathbb{Z}$ with finitely generated kernel.

With this terminology, our main result is:

Theorem 1.2. Let $M$ be a closed three-manifold, and let $\omega \in H^{1}(M ; \mathbb{Z})$ be fully compatible with a handlebody bundle. If $F$ denotes the fibered face whose cone $C_{F}$ contains $\omega$, then every integral class $\omega^{\prime} \in C_{F}$ is fully compatible with some handlebody bundle.

The condition that the inclusion $M \hookrightarrow W$ should induce an isomorphism on $H_{1}$ (required by the definition of full compatibility) is easy to check, and grants us the flexibility to prove the following application.

Theorem 1.3. Suppose that $\Gamma$ is a free-by-cyclic group. Then there are infinitely many pairwise non-diffeomorphic, hyperbolic three-manifolds admitting fibered classes fully compatible with handlebody bundles with fundamental group $\Gamma$.

Finally, we want to emphasise that the theorem yields a new connection between mapping classes of surfaces and (outer) automorphisms of free groups; to every automorphism we can associate (infinitely many different) mapping classes, which should inherit properties from the free group automorphism. To our knowledge, this intriguing connection has not yet been explored.

Acknowledgements. The authors would like to thank the organizers of the "Moduli Spaces" conference on Ventotene in 2017, where some of this work was conducted.

The second author was supported by the grant KI 1853/3-1 within the Priority Programme 2026 "Geometry at Infinity" of the German Science Foundation (DFG). 


\section{The Thurston Polytope For three-MAnifolds And FREE-BY-CYCLIC GROUPS}

Throughout, we will use the notation established in the introduction: $M$ is a closed, connected and oriented three-manifold which fibres over the circle with fiber $S_{g}$, associated class $\omega \in H^{1}(M ; \mathbb{Z})$ and monodromy $\varphi$.

A group will be called free-by-cyclic if it is an extension of a finitely generated free group by $\mathbb{Z}$. This is the case for the fundamental group of every handlebody bundle $W$ with which $\omega$ is compatible.

Thurston [Thu86, Theorem 5] proved that all the different ways in which a given three-manifold can fiber over the circle are encoded by a polytope, in a way which we will make precise in a moment. The second author gave a new proof of Thurston's theorem in [Kie20, Theorem 5.34], and then extended the result to cover also free-by-cyclic groups [Kie20, Theorem 5.29] - in this latter setting, 'fibering over the circle' is interpreted to mean the existence of an epimorphism to $\mathbb{Z}$ with a finitely generated kernel.

Note that, thanks to a result of Stallings [Sta62], an integral cohomology 1-class $\omega: \pi_{1}(M) \rightarrow \mathbb{Z}$ of an irreducible three-manifold $M$ is fibered if and only if ker $\omega$ is finitely generated. (Recall that $\omega$ being fibered means that it is induced by $M$ fibering over the circle.) Moreover, one can remove the assumption of $M$ being irreducible thanks to Perelman's solution of the Poincaré conjecture. Hence, the group-theoretic notion of fibering used above coincides with the topological one for three-manifold groups.

It is important to note that if the kernel of $\omega: G \rightarrow \mathbb{Z}$ is finitely generated (that is, if $\omega$ is fibered), then ker $\omega$ is in fact a surface group or a free group if $G$ is a three-manifold group, and a free group if $G$ is a free-by-cyclic group (the latter by [GMSW01]). In either case, the kernel has a well-defined Euler characteristic, denoted by $\chi(\operatorname{ker} \omega)$.

Before proceeding, let us state some definitions: a polytope in a finitedimensional $\mathbb{R}$-vector space $V$ denotes the intersection of finitely many halfspaces, and therefore a polytope $P$ must be convex, but need not be compact. Unless explicitly stated to the contrary, we will also require polytopes to be symmetric, that is preserved by the map $v \mapsto-v$. Given a face $F \neq\{0\}$ of a polytope, which for us will always mean an open face, we define $C_{F}$ to be the cone over that face; explicitly, we set

$$
C_{F}=\{\lambda v \mid v \in F, \lambda \in(0, \infty)\} .
$$

When $P=F=\{0\}$ we define $C_{F}=V$.

Theorem 2.1 ([Thu86, Kie20]). Suppose that $G$ is a three-manifold group or a free-by-cyclic group. There exists a polytope $P$ in $H^{1}(G ; \mathbb{R})$ such that for every epimorphism $\omega: G \rightarrow \mathbb{Z}$ with a finitely generated kernel there exists a face $F$ (the associated fibered face) of $P$ with $\omega \in C_{F}$ such that

(1) $F$ is top-dimensional, or equivalently, $C_{F}$ is open, and

(2) every primitive integral class $\omega^{\prime} \in H_{1}(G ; \mathbb{Z})$ lying in $C_{F}$ has a finitely generated kernel, and 
(3) the map $\omega^{\prime} \mapsto \chi\left(\operatorname{ker} \omega^{\prime}\right)$ defined on the primitive integral classes in $C_{F}$ extends to a linear functional defined on the whole of $C_{F}$.

Proof. We begin by examining the pathological case of $G=\mathbb{Z}$. Note that $\mathbb{Z}$ is at the same time the fundamental group of a closed three-manifold, and a free-by-cyclic group.

In this case we take $P=\{0\}$. The unique face of $P$ is maximal dimensional, and its cone $V=\mathbb{R}$ is open. There are exactly two primitive integral classes, and their kernels are the trivial group, which certainly is finitely generated. The functional $v \mapsto|v|$ extends the map $\omega^{\prime} \mapsto \chi\left(\operatorname{ker} \omega^{\prime}\right)=1$. In what follows we will assume that $G \neq \mathbb{Z}$.

Let us start from the more classical case, in which $G$ is a three-manifold group which is not $\mathbb{S}^{2} \times \mathbb{S}^{1}$. The polytope $P$ above is what is denoted by $B_{x}$ in $[\mathrm{Thu} 86]$, and is the unit ball of the Thurston norm $x: H^{1}(G ; \mathbb{R}) \rightarrow$ $[0, \infty)$. The Thurston norm $x\left(\omega^{\prime}\right)$ of a primitive cohomology class $\omega^{\prime} \in$ $H^{1}(G ; \mathbb{Z})$ with finitely generated kernel is equal to $-\chi\left(\operatorname{ker} \omega^{\prime}\right)$ by definition. For convenience, we define $N=\frac{1}{2} x: H^{1}(G ; \mathbb{R}) \rightarrow[0, \infty)$.

It is immediate that $x$, and hence $N$, are linear on $C_{F}$.

The facts that $C_{F}$ is open and that every primitive integral class therein is fibered follow from [Thu86, Theorems 3 and 5].

Now suppose that $G$ is a free-by-cyclic group, where the free kernel is not trivial. The starting point is the $L^{2}$-torsion polytope $P_{L^{2}} \subseteq H_{1}(G ; \mathbb{R})$ appearing in [Kie20, Theorem 5.29], and introduced first by Friedl-Lück [FL17]. Note that $P_{L^{2}}$ is in general not symmetric. The polytope $P_{L^{2}}$ induces a thickness function $T: H^{1}(G ; \mathbb{R}) \rightarrow[0, \infty)$ by setting

$$
T\left(\omega^{\prime}\right)=\max _{p, q \in P_{L^{2}}}\left|\omega^{\prime}(p)-\omega^{\prime}(q)\right|
$$

In fact, $T$ is a semi-norm by [FK18, Corollary 3.5], and if $\operatorname{ker} \omega^{\prime}$ is finitely generated and $\omega^{\prime}$ is primitive, then

$$
T\left(\omega^{\prime}\right)=-\chi\left(\operatorname{ker} \omega^{\prime}\right)
$$

by the proof of [FK18, Theorem 4.4] (see also [HK, Theorem 6.2 and Remark $6.5])$.

The desired polytope $P$ is defined to be the unit ball of the semi-norm $T$. This immediately implies that $T$ is linear on the cones of the faces of $P$. Note that, in general $P$ is not the same as $P_{L^{2}}$. In particular, $P$ is symmetric, whereas $P_{L^{2}}$ does not have to be.

Since ker $\omega$ is finitely generated, we have $\omega$ and $-\omega$ lying in the (first) Bieri-Neumann-Strebel invariant $\Sigma^{1}(G)$ by [BNS87, Theorem B1], and therefore [Kie20, Theorem 5.29] tells us that there are unique points $p$ and $q \in P_{L^{2}}$ such that $\omega$ restricted to $P_{L^{2}}$ attains its minimum at $p$ and maximum at $q$. But this is an open condition, and therefore $T$ is linear on a neighbourhood $U$ of $\omega$. This implies that the cone $C_{F}$ containing $\omega$ contains $U$, where $F$ is a face of $P$. Hence $C_{F}$ has non-empty interior. This implies that $F$ is maximal dimensional, and hence $C_{F}$ is open. 
The cone $C_{F}$ consists of precisely these cohomology classes which, when restricted to $P_{L^{2}}$, attain their minimum precisely at $p$ and their maximum precisely at $q$. Therefore, every integral class in $C_{F}$ is fibered by [Kie20, Theorem 5.29].

\section{All Fiberings are Handlebody}

In this section we assume in addition to the assumptions of the last section that $\omega$ is fully compatible with a handlebody bundle $W$ which fibers with fiber a handlebody $V_{g}$. We also let $F$ denote the fibered face of $P_{M}$, the Thurston polytope, whose cone $C_{F}$ contains $\omega$. We set $\Gamma=\pi_{1}(W)$ as before.

As indicated in the introduction, we have the following diagram with exact rows:

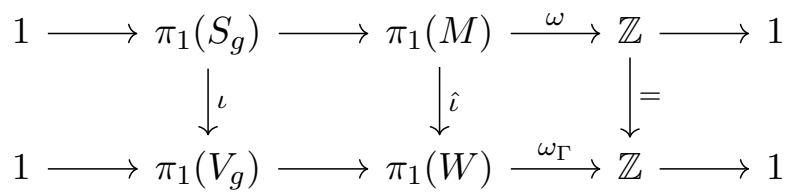

Here $\iota, \hat{\iota}$ are the maps induced by the inclusions of the boundary. Note that since $\iota$ is surjective, so is $\hat{\imath}$.

Recall that we are also assuming that the epimorphism $\hat{\iota}$ induces an isomorphism

$$
\hat{\iota}_{*}: H_{1}(M ; \mathbb{Q}) \rightarrow H_{1}(W ; \mathbb{Q}) .
$$

Let $F_{k}$ denote the free group of rank $k$. We need the following ingredient:

Proposition 3.1 (Co-rank theorem for surface groups). If $f: \pi_{1}\left(S_{g}\right) \rightarrow F_{k}$ is a surjective map, then $k \leqslant g$. In the case of equality, the map $f$ is induced by the identification of $S_{g}$ with the boundary of a genus $g$ handlebody $V_{g}$. Furthermore, if in that case $\psi$ is any mapping class of $S_{g}$ which preserves $\operatorname{ker}(f)$, then $\psi$ has an extension to $V_{g}$.

Proof. The fact that $k \leqslant g$ is [LR02, Lemma 2.1], while the fact on the identification with a handlebody is [LR02, Lemma 2.2]. The fact that any mapping class of $\partial V_{g}$ which preserves $\operatorname{ker}\left(\pi_{1}\left(\partial V_{g}\right) \rightarrow \pi_{1}\left(V_{g}\right)\right)$ extends to the handlebody $V$ is standard, see e.g. [Hen17, Corollary 5.11].

We are now ready to prove the main theorem.

Proof of Theorem 1.2. Let $\omega^{\prime}: \pi_{1}(M) \rightarrow \mathbb{Z}$ be an epimorphism lying in the cone $C_{F}$. Since $\pi_{1}(M) \rightarrow \pi_{1}(W)$ is surjective, and induces an isomorphism $H_{1}(M ; \mathbb{Q}) \cong H_{1}(W ; \mathbb{Q})$, there is an epimorphism $\omega_{\Gamma}^{\prime}$ that makes the right square in the following diagram commute: 


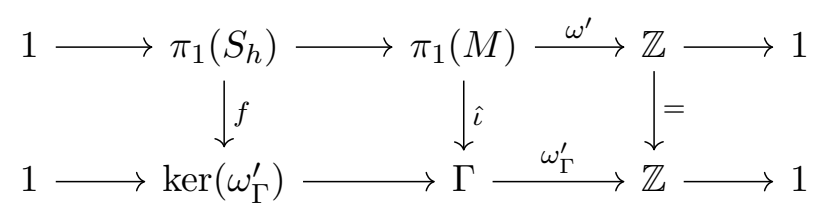

By a simple diagram chase, a homomorphism $f$ which makes the left square commute exists, and is surjective. Therefore, $H=\operatorname{ker}\left(\omega_{W}^{\prime}\right)$ is finitely generated. But $\Gamma=\pi_{1}(W)$ is a free-by-cyclic group, and hence $H$ is a free group by [GMSW01].

We now claim that the rank of $H$ is at least $h$. Suppose first that we have shown the claim. Now the co-rank theorem for surface groups (Proposition 3.1) tell us that the rank is exactly $h$. Let $x \in \operatorname{ker} f$, and let $z \in \pi_{1}(M)$ denote some preimage under $\omega^{\prime}$ of a generator of $\mathbb{Z}$. We have

$$
f\left(z^{-1} x z\right)=\hat{\iota}\left(z^{-1} x z\right)=\hat{\iota}\left(z^{-1}\right) \hat{\iota}(x) \hat{\iota}(z)=\hat{\imath}\left(z^{-1}\right) f(x) \hat{\iota}(z)=1
$$

and so ker $f$ is preserved by the monodromy induced by $\omega^{\prime}$ (whose action coincides with conjugation by $z$ ). The second part of the co-rank theorem now gives us a homeomorphism of the corresponding handlebody $V_{h}$ with boundary $S_{h}$ extending the monodromy induced by $\omega^{\prime}$.

We are left with proving the claim. For a contradiction, suppose that the rank $g$ of $H$ is strictly smaller than $h$. Write $v=\omega_{\Gamma}-\omega_{\Gamma}^{\prime}$; we then have $\hat{\iota}^{*} v=\omega-\omega^{\prime}$. Observe that by Theorem 2.1, there are nondegenerate linear functionals $N$ (half of the Thurston norm) and $T$ (the thickness function), such that

$$
g-h=\frac{1}{2}\left(\chi\left(S_{h}\right)-\chi\left(S_{g}\right)\right)=N\left(\hat{\iota}^{*}(v)\right)
$$

and

$$
\operatorname{rk}\left(\operatorname{ker}\left(\omega_{\Gamma}\right)\right)-\operatorname{rk}\left(\operatorname{ker}\left(\omega_{\Gamma}^{\prime}\right)\right)=\chi\left(\operatorname{ker}\left(\omega_{\Gamma}^{\prime}\right)\right)-\chi\left(\operatorname{ker}\left(\omega_{\Gamma}\right)\right)=T(v)
$$

Since $g=\operatorname{rk}\left(\operatorname{ker}\left(\omega_{\Gamma}\right)\right)$, and $\operatorname{rk}\left(\operatorname{ker}\left(\omega_{\Gamma}^{\prime}\right)\right)<h$, this implies

$$
N\left(\hat{\iota}^{*}(v)\right)<T(v) .
$$

Consider $\omega_{\Gamma}^{\prime \prime}=\omega_{\Gamma}+q v$ and $\omega^{\prime \prime}=\omega+q \hat{\iota}^{*} v$ for a small rational number $q$. Since $q$ is rational, the cohomology class $\omega^{\prime \prime}$ is also rational, in the sense that $\omega^{\prime \prime} \in H^{1}(M ; \mathbb{Q})$. There exists a unique positive integer $k$ such that $k \omega^{\prime \prime}$ is integral and primitive. Also, since $q$ is small, $k \omega^{\prime \prime}$ lies in the cone of the same fibered face as $\omega$, and hence is a fibered character. Arguing as before using Proposition 3.1 and [GMSW01], we thus have

$$
T\left(\omega_{\Gamma}^{\prime \prime}\right)=\frac{-1}{k} \chi\left(\operatorname{ker}\left(k \omega_{\Gamma}^{\prime \prime}\right)\right) \leqslant \frac{-1}{2 k} \chi\left(\operatorname{ker}\left(k \omega^{\prime \prime}\right)\right)=N\left(\omega^{\prime \prime}\right)
$$

We also have

$$
N\left(\omega^{\prime \prime}\right)=N(\omega)+q N\left(\hat{\iota}^{*} v\right)<T\left(\omega_{\Gamma}\right)+q T(v)=T\left(\omega_{\Gamma}^{\prime \prime}\right)
$$


and so

$$
T\left(\omega_{\Gamma}^{\prime \prime}\right) \leqslant N\left(\omega^{\prime \prime}\right)<T\left(\omega_{\Gamma}^{\prime \prime}\right)
$$

which is a contradiction. We have therefore proven the claim.

\section{Existence of FUlly COMPATIBLE FIBERED ClASSES}

In this section we show how to construct bundles $M$ that will satisfy the assumption that there exists an isomorphism $\hat{\iota}_{*}: H_{1}(M ; \mathbb{Q}) \cong H_{1}(W ; \mathbb{Q})$ where $\pi_{1}(W)$ is a given free-by-cyclic group. More precisely, we will show the following, which is a rephrasing of Theorem 1.3.

Theorem 4.1. Given any free group automorphism $f: F_{g} \rightarrow F_{g}$, there are mapping classes $\varphi_{i}$ of the handlebody $V_{g}$ such that

i) $\varphi_{i}$ induces the automorphism $f$ on $\pi_{1}\left(V_{g}\right)=F_{g}$ for all $i$.

ii) The (four-manifolds) $W_{i}$ obtained as the mapping tori of the mapping classes $\varphi_{i}$ satisfy $\hat{\iota}_{*}: H_{1}\left(\partial W_{i} ; \mathbb{Q}\right) \cong H_{1}\left(W_{i} ; \mathbb{Q}\right)$ for all $i$.

iii) The (three-manifolds) $M_{i}=\partial W_{i}$ are hyperbolic for all $i$ and are pairwise non-diffeomorphic.

Before we can give the proof, we need some basic notation. Recall that if $S_{g}$ is a closed surface of genus $g$, the algebraic intersection number defines a symplectic pairing

$$
H_{1}\left(S_{g} ; \mathbb{Z}\right) \times H_{1}\left(S_{g} ; \mathbb{Z}\right) \rightarrow \mathbb{Z}
$$

which extends in the obvious way to

$$
\sigma: H_{1}\left(S_{g} ; \mathbb{Q}\right) \times H_{1}\left(S_{g} ; \mathbb{Q}\right) \rightarrow \mathbb{Q}
$$

on the first homology group. Suppose now that $S_{g}$ has been identified with the boundary $\partial V_{g}$ of a handlebody. Then, the inclusion of the boundary defines a map

$$
\iota_{*}: H_{1}\left(S_{g} ; \mathbb{Q}\right) \rightarrow H_{1}\left(V_{g} ; \mathbb{Q}\right)
$$

whose kernel we denote by $L$. Explicitly, let $\alpha_{1}, \ldots, \alpha_{g}$ be disjoint curves bounding disks which cut the handlebody $V_{g}$ into a ball. Choose curves $\beta_{i}$ with the property that $\sigma\left(\alpha_{i}, \beta_{j}\right)$ is 0 if $i \neq j$ and 1 otherwise. Then the homology classes $a_{i}, b_{j}$ defined by the curves $\alpha_{i}, \beta_{j}$, respectively, are a basis for $H_{1}\left(S_{g} ; \mathbb{Q}\right)$. We then have that

$$
L=\operatorname{ker}\left(\iota_{*}\right)=\left\langle a_{1}, \ldots, a_{g}\right\rangle .
$$

Furthermore, if we define

$$
D=\left\langle b_{1}, \ldots, b_{g}\right\rangle,
$$

then the restriction $\iota_{*}: D \rightarrow H_{1}\left(V_{g} ; \mathbb{Q}\right)$ is an isomorphism. Furthermore, $\sigma$ vanishes identically on $L$ and $D$. In other words, we have

$$
H_{1}\left(S_{g} ; \mathbb{Q}\right)=L \oplus D,
$$


and both $L, D$ are Lagrangian subspaces. With respect to this decomposition, $\sigma$ corresponds to the matrix

$$
J=\left(\begin{array}{cc}
0 & \mathrm{Id} \\
-\mathrm{Id} & 0
\end{array}\right)
$$

Denote by $\mathcal{H}_{g}<\operatorname{Mcg}\left(S_{g}\right)$ the handlebody group, i.e. the subgroup of those mapping classes of $S_{g}$ which extend to $V_{g}$. If $\phi$ is an element of the handlebody group, then $\phi_{*}(L)=L$. This gives the following obstruction for how the handlebody group acts on homology.

Lemma 4.2 (e.g. [Bir75, Lemma 2.2]). For a symplectic basis as above, every handlebody group element $\phi$ acts on $H_{1}\left(S_{g} ; \mathbb{Q}\right)$ as a matrix of the form

$$
\phi_{*}=\left(\begin{array}{cc}
A & B \\
0 & \left(A^{t}\right)^{-1}
\end{array}\right),
$$

where $A$ is invertible, $B$ satisfies $B^{t}\left(A^{t}\right)^{-1}=A^{-1} B$, and the entries are all integers. Conversely, any such matrix is realised as the action of a suitable handlebody group element $\phi$.

Furthermore, $\left(A^{t}\right)^{-1}$ is the matrix representing the action of $\phi$ on $H_{1}\left(V_{g}, \mathbb{Q}\right)$ with respect to the basis $b_{1}, \ldots, b_{g}$.

We also need the following variant, which is likely well-known to experts.

Lemma 4.3. For a basis of $H_{1}\left(S_{g} ; \mathbb{Q}\right)$ as above, every integral symplectic matrix of the form

$$
\left(\begin{array}{cc}
\mathrm{Id} & B \\
0 & \mathrm{Id}
\end{array}\right)
$$

can be realised as the homology action of a handlebody mapping class which acts trivially on the fundamental group of the handlebody.

Proof. The condition that the matrix is symplectic implies that $B$ has to be symmetric. First, let $i$ be given. The twist about $\alpha_{i}$ acts as the matrix

$$
\left(\begin{array}{cc}
\text { Id } & E_{i} \\
0 & \text { Id }
\end{array}\right)
$$

where $E_{i}$ is the matrix which is zero, except a single diagonal entry 1 in column $i$.

Next, let $i \neq j$ be given. Let $\delta$ be a diskbounding curve which intersects each of $\beta_{i}, \beta_{j}$ in a single point, and defines the homology class $a_{i}+a_{j}$. The twist about $\delta$ acts as

$$
\left(\begin{array}{cc}
\mathrm{Id} & E_{i}+E_{j}+E_{i j}+E_{j i} \\
0 & \mathrm{Id}
\end{array}\right)
$$

where $E_{i j}$ is the elementary matrix with entry 1 in row $i$, column $j$. Since Dehn twists about diskbounding curves extend to the handlebody, and their extensions act trivially on the fundamental group of the handlebody, the lemma is proved for matrices of the form $B=E_{i}$ and $B=E_{i j}+E_{j i}$. Since 
these (additively) generate the group of integral symmetric matrices, the lemma follows.

To certify that $\hat{\iota}_{*}: H_{1}\left(M_{i} ; \mathbb{Q}\right) \cong H_{1}\left(W_{i} ; \mathbb{Q}\right)$ in the proof of Theorem 4.1, we will use the following criterion.

Lemma 4.4. Suppose that $\phi$ is a handlebody group element, and let $A, B$ be the blocks of $\phi_{*}$ as in Lemma 4.2. If

$$
\operatorname{im}(A-\mathrm{Id})+B\left(\operatorname{ker}\left(\left(A^{t}\right)^{-1}-\mathrm{Id}\right)\right)=L,
$$

then the handlebody bundle $W$ obtained as the mapping torus of $\phi$ satisfies $\hat{\iota}_{*}: H_{1}(M ; \mathbb{Q}) \cong H_{1}(W ; \mathbb{Q})$ where $M=\partial W$.

Proof. We have

$$
H_{1}(M ; \mathbb{Q})=\mathbb{Q} \oplus\left(H_{1}\left(S_{g} ; \mathbb{Q}\right)\right) /\left(\operatorname{Id}-\phi_{*}\right)=\mathbb{Q} \oplus(L \oplus D) /\left(\operatorname{Id}-\phi_{*}\right) .
$$

By assumption, we have

$$
L=\operatorname{im}(A-\mathrm{Id})+B\left(\operatorname{ker}\left(\left(A^{t}\right)^{-1}-\mathrm{Id}\right)\right)
$$

Hence, the natural map

$$
\mathbb{Q} \oplus D /\left(\operatorname{Id}-\left(A^{t}\right)^{-1}\right) \rightarrow \mathbb{Q} \oplus(L \oplus D) /\left(\operatorname{Id}-\phi_{*}\right)
$$

is surjective, and it is clearly injective. On the other hand, if we denote by $f_{*}: \pi_{1}(V) \rightarrow \pi_{1}(V)$ the map induced by the handlebody group element $\phi$, we have

$$
H_{1}(W ; \mathbb{Q})=\mathbb{Q} \oplus\left(H_{1}\left(V_{g} ; \mathbb{Q}\right) /\left(\operatorname{Id}-f_{*}\right)\right) \cong \mathbb{Q} \oplus D /\left(\operatorname{Id}-\left(A^{t}\right)^{-1}\right) .
$$

Together, these imply the lemma.

We are now ready to begin the proof of Theorem 4.1 in earnest.

Proof of Theorem 4.1. Let $f: F_{g} \rightarrow F_{g}$ be given. Up to replacing $f$ by a conjugate, we may assume that $f$ acts on homology as

$$
f_{*}=\left(\begin{array}{cc}
\mathrm{Id} & U \\
0 & V
\end{array}\right)
$$

where $V$ does not have any eigenvalue 1 . This follows since $\operatorname{Out}\left(F_{n}\right) \rightarrow$ $\mathrm{GL}_{n}(\mathbb{Z})$ is surjective, and integral matrices can be (integrally) conjugated to have this form.

Since the map $\mathcal{H}_{g} \rightarrow \operatorname{Out}\left(F_{g}\right)$ is also surjective (e.g. [Gri63]) and the claims in Theorem 4.1 are invariant under replacing $\left(\varphi_{i}\right)$ by $\left(\psi \varphi_{i} \psi^{-1}\right)$, it suffices to show the theorem under this assumption on $f_{*}$.

Now, take a handlebody group element $\phi$ which acts as $f$ on $\pi_{1}(V)$. Let $A$ be the matrix satisfying $\left(A^{t}\right)^{-1}=f_{*}$.

Lemma 4.5. With notation and assumptions as above, there is a matrix $B$ such that

and $B^{t}\left(A^{t}\right)^{-1}=A^{-1} B$.

$$
\operatorname{im}(A-\mathrm{Id})+B\left(\operatorname{ker}\left(\left(A^{t}\right)^{-1}-\mathrm{Id}\right)\right)=L,
$$


Proof. Under the assumptions, we have

$$
A^{t}=\left(\begin{array}{cc}
\mathrm{Id} & Y \\
0 & Z
\end{array}\right)
$$

where $Z$ is a $k \times k$ matrix such that $Z$ - Id is injective, and $Y$ is a $(g-k) \times k$ matrix. In particular, $Z-\operatorname{Id}$ is invertible over $\mathbb{Q}$.

We then have

$$
A=\left(\begin{array}{cc}
\mathrm{Id} & 0 \\
Y^{t} & Z^{t}
\end{array}\right)
$$

and observe that thus the image $(A-\operatorname{Id})\left(\mathbb{Q}^{2 g}\right)$ is the subspace spanned by the last $k$ basis vectors. Put

$$
B=\left(\begin{array}{ll}
\operatorname{Id} & 0 \\
Y^{t} & 0
\end{array}\right) .
$$

Observe that $\operatorname{ker}\left(\left(A^{t}\right)^{-1}-\mathrm{Id}\right)=\operatorname{ker}\left(A^{t}-\mathrm{Id}\right)$, and therefore it is the subspace spanned by the first $g-k$ basis vectors. Together, this implies that $\operatorname{im}(A-\mathrm{Id})$ and $B\left(\operatorname{ker}\left(\left(A^{t}\right)^{-1}-\mathrm{Id}\right)\right)$ span $L$. In other words, $B$ satisfies

$$
\operatorname{im}(A-\mathrm{Id})+B\left(\operatorname{ker}\left(\left(A^{t}\right)^{-1}-\mathrm{Id}\right)\right)=L,
$$

as claimed.

Furthermore, we have

$$
A B^{t}=\left(\begin{array}{cc}
\operatorname{Id} & Y \\
Y^{t} & Y^{t} Y
\end{array}\right)=B A^{t}
$$

Now, let $B$ be a matrix as given by Lemma 4.5. Since $\mathcal{H}_{g} \rightarrow \operatorname{Out}\left(F_{g}\right)$ is surjective, we can find a handlebody group element $\phi$ mapping to $f$. It then acts on $H_{1}(S ; \mathbb{Q})$ as

$$
\left(\begin{array}{cc}
A & B^{\prime} \\
0 & \left(A^{t}\right)^{-1}
\end{array}\right)
$$

since the lower right block describes the action on the first homology of the handlebody. Applying Lemma 4.3, we can therefore find a handlebody group element $\varphi_{0}$ which acts as

$$
\left(\begin{array}{cc}
A & B \\
0 & \left(A^{t}\right)^{-1}
\end{array}\right)
$$

By construction of $B$ and Lemma 4.4, the mapping torus $W_{0}$ defined by $\varphi_{0}$ satisfies conditions i) and ii) of Theorem 4.1. Now let $\psi$ be an element of the kernel of the map

$$
\mathcal{H}_{g} \rightarrow \operatorname{Out}\left(F_{g}\right)
$$

such that $\left.\psi\right|_{\partial V_{g}}$ is pseudo-Anosov and such that $\psi$ acts as the identity on $H_{1}\left(\partial V_{g} ; \mathbb{Q}\right)$. Such a mapping class can for example be constructed as the product of two Dehn twists about separating curves bounding disks.

Observe that for all integers $n$, the mapping tori defined by the elements $\psi^{n} \varphi_{0}$ then also satisfy i) and ii), since they act on $H_{1}(S ; \mathbb{Q})$ in the same way as $\varphi_{0}$. On the other hand, for large $n$, the elements $\left.\psi^{n} \varphi_{0}\right|_{\partial V_{g}}$ are pseudo-Anosov with diverging Weil-Petersson translation length. Thus, the 
mapping tori defined by the boundary maps of $\psi^{n} \varphi_{0}$ are hyperbolic manifolds, and by the main theorem of [Bro03] their volumes diverge. By Mostow rigidity this implies in particular that there are infinitely many distinct diffeomorphism classes in the sequence.

This shows Theorem 4.1.

\section{REFERENCES}

[Bir75] J. S. Birman, On the equivalence of Heegaard splittings of closed, orientable 3-manifolds (1975), 137-164. Ann. of Math. Studies, No. 84. MR0375318

[BNS87] R. Bieri, W. D. Neumann, and R. Strebel, A geometric invariant of discrete groups, Invent. Math. 90 (1987), no. 3, 451-477. MR914846 (89b:20108)

[Bro03] J. F. Brock, Weil-Petersson translation distance and volumes of mapping tori, Comm. Anal. Geom. 11 (2003), no. 5, 987-999. MR2032506

[DKL15] S. Dowdall, I. Kapovich, and C. J. Leininger, Dynamics on free-by-cyclic groups, Geom. Topol. 19 (2015), no. 5, 2801-2899. MR3416115

[DKL17a] _ Endomorphisms, train track maps, and fully irreducible monodromies, Groups Geom. Dyn. 11 (2017), no. 4, 1179-1200. MR3737279

[DKL17b] - McMullen polynomials and Lipschitz flows for free-by-cyclic groups, J. Eur. Math. Soc. (JEMS) 19 (2017), no. 11, 3253-3353. MR3713041

[FK18] F. Funke and D. Kielak, Alexander and Thurston norms, and the BieriNeumann-Strebel invariants for free-by-cyclic groups, Geom. Topol. 22 (2018), no. 5, 2647-2696. MR3811767

[FL17] S. Friedl and W. Lück, Universal $L^{2}$-torsion, polytopes and applications to 3-manifolds, Proc. Lond. Math. Soc. (3) 114 (2017), no. 6, 1114-1151. MR3661347

[GMSW01] R. Geoghegan, M. L. Mihalik, M. Sapir, and D. T. Wise, Ascending HNN extensions of finitely generated free groups are Hopfian, Bull. London Math. Soc. 33 (2001), no. 3, 292-298. MR1817768 (2002a:20029)

[Gri63] H. B. Griffiths, Automorphisms of a 3-dimensional handlebody, Abh. Math. Sem. Univ. Hamburg 26 (1963/1964), 191-210. MR0159313

[Hen17] S. Hensel, A primer on handlebody groups, preprint (2017). available at http://www . mathematik. uni-muenchen.de/ hensel.

[HK] F. Henneke and D. Kielak, Agrarian and $L^{2}$-invariants, arXiv:1809.08470.

[Kie20] D. Kielak, The Bieri-Neumann-Strebel invariants via Newton polytopes, Invent. Math. 219 (2020), no. 3, 1009-1068. MR4055183

[LR02] C. J. Leininger and A. W. Reid, The co-rank conjecture for 3-manifold groups, Algebr. Geom. Topol. 2 (2002), 37-50. MR1885215

[Mut] J. P. Mutanguha, Irreducibility of a free group endomorphism is a mapping torus invariant, arXiv:1910.04285.

[Sta62] J. Stallings, On fibering certain 3-manifolds, Topology of 3-manifolds and related topics (Proc. The Univ. of Georgia Institute, 1961), 1962, pp. 95-100. MR0158375

[Thu86] W. P. Thurston, A norm for the homology of 3-manifolds, Mem. Amer. Math. Soc. 59 (1986), no. 339, i-vi and 99-130. MR823443 (88h:57014)

Sebastian Hensel

hensel@math.lmu.de

Mathematisches Institut

der Universität München

Theresienstraße 39
Dawid Kielak

dkielak@math.uni-bielefeld.de Fakultät für Mathematik Universität Bielefeld Postfach 100031 
80333 München

Germany
33501 Bielefeld

Germany 